\title{
INFORMATION
}

\section{RESEARCH METHODS IN INTERCULTURAL COMMUNICATION. A PRACTICAL GUIDE}

\author{
Edited by Zhu Hua \\ Wiley Blackwell, 2015 \\ Nguyen Thi Thuy Linh
}

Department of English, VNU University of Languages and International Studies, Pham Van Dong, Cau Giay, Hanoi, Vietnam

Received 24 May 2019

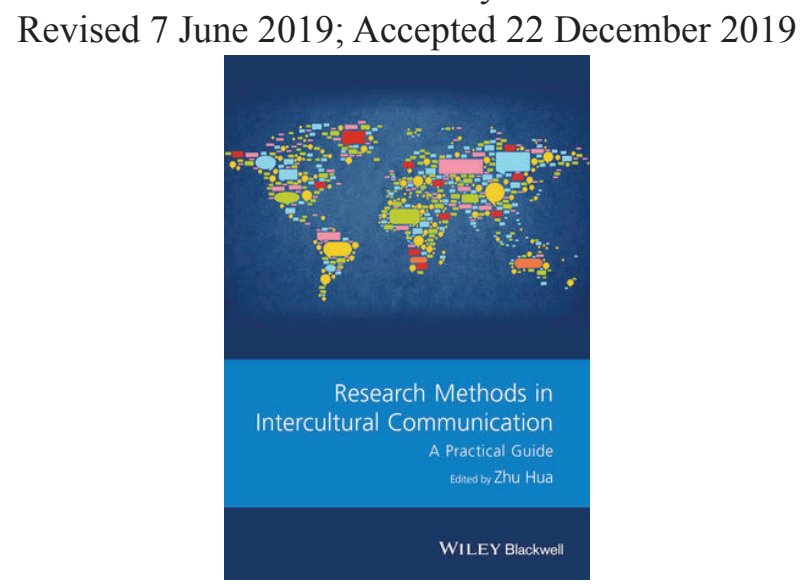

\section{Introduction}

As the latest one in the Wiley Blackwell series "Guides to research methods in language and linguistics", "Research methods in intercultural communication", edited by Zhu Hua, is described on their website as "the most comprehensive volume on research methods in intercultural communication research in the last 30 years" (Wiley, 2016). This book is the contributory collection of a global team of experienced scholars and researchers from intercultural communication, anthropology, education, applied linguistics, and communication, explain both established and emerging research methods and analytical tools. It seeks to provide an introduction to the key methodological issues and concerns in the study of Intercultural Communication for students on advanced undergraduate and postgraduate programs in Intercultural Communication, language and linguistics, applied linguistics, TESOL, education, translation, communication studies and other related subjects.

\section{Contents of the book}

The volume does not start with describing methods right away. Rather, it opens with two parts that often receive little attention in research training but have significant bearings on the validity of research questions and the 
interpretation of results. In brief, the core contents of the book are divided into three main sections, which are I. Linking themes, paradigms, and methods, II. Key issues and challenges and III. Methods.

Part 1 forwards key concepts in understanding intercultural communication. In the first place, an overview of the five key research paradigms in Intercultural studies are provided. Zhu Hua, the editor of this volume starts with confirming the multidisciplinary nature of Intercultural Communication as a field of enquiry. Then, she continues to discuss what a paradigm is and why it is essential to understand paradigms before carrying on research designs. Five paradigms, namely positivist, interpretative, critical, constructivist, and realist are introduced afterwards in terms of their main assumptions, research themes, and disciplinary connections. The next three chapters deals with three key topics in the study of Intercultural Communication: culture, identity and discourse.

In "Studying culture" chapter, Adrian Holliday comes up with a social action "grammar" of culture to indicate the different and interconnected forces that act on culture and Intercultural Communication and provide different foci for research. He concludes, underlying universal cultural processes imply that all of us are equally engaged in the everyday construction of and engagement with culture wherever it is found.

Following is the chapter discussing identity. In this, a brief overview of some key theoretical issued related to the study of identity in the context of different schools of thought, particularly positive and constructivism is provided. Jo Angouri in the end, comes to the conclusion that the term "identity" has become so extended that it can stand for a range of other more or less notion, similarly to culture.

The next chapter brings about the topic of studying discourse. It aims to explore the history of academic fields that focus on analyzing these complex communicative interactions. The second half of the chapter looks at four different methods of discourse analysis: Dell Hymes's SPEAKING model; Labov and Waletzky's Narrative Analysis method; Scollon, Scollon, and Jones's Discourse systems approach; and Leila Monaghan's HISTORY model. The strengths and limitations of each model are discussed, giving students and researchers insights into how these methods might be used in their own work.

After a brief theoretical discussion of key topics in Intercultural Communication, Part II discusses the key issues and challenges in research strategies, planning and implementation, including research questions, researching multilingually, interculturally, and ethically, myths and challenges in measuring intercultural competence, the researcher's role, and a step-by-step guide to developing a research proposal.

Identifying research questions has never been an easy job and indeed, it is a process that requires a series of actions and steps. The nine scenarios identified in the chapter range from "not knowing where to start" to "having too many ideas"; from "questions first", where one starts with a research question to "data first, theory or method first", where one knows what data, theory, or a data collection method they would like to work with prior to a search question.

In the next two chapters, the issues of how to research multilingually, culturally and ethically are elaborated on. They are 
aimed to develop researchers' awareness and understanding of the process of researching interculturally. The seventh chapter discusses the implications of taking what might be considered a cross-cultural as opposed to an intercultural approach. While the first involves a more concrete approach to culture as behavior which can be identified and described, the second involves an understanding of culture as created within interaction.

Chapter 8 explores guiding principles in using measures to assess intercultural competence. It also discusses other key issues in assessment of intercultural competence including the lifelong process of developing intercultural competence, the need to assess behavior, and using collected assessment information for further intercultural development.

In the following, chapter 9, "How to work with research participants: the researcher's role" proposes to "reeducate" the researcher of interculturality. In order to do so, Fred Dervin argues that critical reflexity is essential in all aspects of research.

The last chapter in the second part deals with "How to develop a research proposal". It begins by exploring ways to identify and shape a research idea or problem into a proposal for a project that can contribute to knowledge in this field of study. In the sections that follow, step-by-step guidance through the development of a project proposal is shown and discussed.

Ultimately, the third part delves into providing a concise summary and explain the key features of twelve most common methods in Intercultural Communication. Each chapter addresses the questions of what the method is about, why this method and why not (strengths and limitations), how to do it, what research themes this method is associated with, how it works with other methods, and what are the new and emerging data-collection and analysis methods and tools. The twelve most common research methods are: questionnaires and surveys, interviews, the matched-guise technique, discourse completion tasks, the critical incident technique, ethnography, virtual ethnography, multimodality, critical discourse analysis, conversation analysis, corpus analysis and narrative analysis.

\section{Discussion}

The journey of the book has brought along some useful understanding for the readers towards the topic of Intercultural communication studies. Within the length of approximately 350 pages, the book contains such a great deal of notable features that makes itself unique and worth reading.

First of all, it is a wealth of literature related to intercultural studies. Starting from the very first part in research paradigms, the authors have pulled near something farreaching and always theoretical towards the readers by supplying the very up-to-date and concise summary of each paradigms. Issues are discussed in a thorough and enchanting way, then examples follow to clarify prior points. An interesting feature in this book is the "case in point", which provides reliable and exemplary research to illustrate a certain subject or topic. The key terms appearing during the discussion of every section are given and explained succinctly with an aim to make them more accessible and easier to remember. What is more, along with the great body of knowledge and theories, the list of references, further reading and resources are also impressive. Many important and prevailing research works have been mentioned and categorized so that the readers can find and read extensively. 
Secondly, it is undeniable that the book has supplied the readers with a userfriendly and practical guide to approach the seemingly hard-to-digest research methods in Intercultural Communication. The act of conducting a research has been summarized in several key steps such as "How to identify research questions" or "How to develop a research proposal". The authors choose to approach the issue from the perspective of an undergraduate student who is struggling with writing up an intercultural communication study. Many obstacles have been tackled and afterwards a great of advice from experts has been provided. Besides that, the list of twelve most common research methods dominating intercultural studies is of great use. The way authors present method by method and briefly discussing its strengths and weaknesses may assist the readers better choose their appropriate methods for their own study.

However, beside all the plus points that ZhuHua has managed to compile and deliver, there are still some recommendations to enhance the quality of this book.

In terms of the third part about twelve most common research methods in intercultural communication, it is agreed that the group of authors have presented a very clear, brief and easy to understand picture of each and every method. However, it is actually rather short and within the length of 20-30 pages per method, the authors just stop at explaining the key general points such as what the method is about, when it is appropriate to use it, and when it is not, some advice about good practice in applying the method, an analysis of strengths and weaknesses of the method, and finally some suggestions for areas in which the method could be applied to intercultural communication method. Perhaps the space is rather limited, thus only in some methods such as ethnography, virtual ethnography and multimodality the development of the research method is covered. This can be viewed as an inconsistency concerning the format and content of all the methods from questionnaires and surveys to narrative analysis. For the students who want to apply a certain method in their studies or theses, reading this book is not enough since it somehow presents the introductory parts of the research methods. Thus, they sure need a lot of time and hard work on delving into the list of recommended readings at the end of every chapter.

Another remark is on the fact that after every chapter in the book, the authors recap and review the discussed knowledge by a short conclusion. In this way, the readers can see the overall picture of what they have just read above and sometimes the conclusion helps trigger more ideas and better understanding of the issue in point. At the end of the third part on methods, it is expected one final conclusion that draws together the themes, key topics and purpose of the volume may come out. Contrary to that thought, none exists and the book is forced to stop in an abrupt way to move to appendixes and index.

Reflecting on the volume as a whole, I still highly regard it as a particularly distinguishing and in deed a must-have item for any students who wish to investigate into intercultural communication studies. The group of authors and especially Zhu Hua as the editor have done such a great job in succeeding to make the broad field of research become something practical, accessible and precise. It can be a useful tool for undergraduates in many fields of linguistics to gain deeper understanding in intercultural communication and dominant methods. Otherwise, research supervisors may find it as a handbook of great help in supplying their students with the appropriate method or approach in line with their research questions. 